\title{
Malignant Peritoneal Mesothelioma with Latent Tuberculosis Infection
}

\author{
Naonori Inoue, Kei Takumi, Daiki Sone, Atsushi Sirakawa, Takuji Kawamura and Koji Uno
}

\begin{abstract}
:
Malignant peritoneal mesothelioma (MPM) is a rare malignant tumor with peritoneal thickening. Tuberculous peritonitis also shows peritoneal thickening, so differentiating between the two is important but difficult if latent tuberculosis infection (LTBI) is present. We herein report a patient with MPM and LTBI. A 79-yearold man was diagnosed with peritoneal thickening on computed tomography. Interferon gamma release assay (IGRA) results were positive, suggesting tuberculous peritonitis. He underwent a laparoscopic omental biopsy and was diagnosed with MPM, which can occur together with LTBI. If peritoneal thickening is observed, an IGRA should be performed early, and the possibility of LTBI should be considered.
\end{abstract}

Key words: malignant peritoneal mesothelioma, latent tuberculosis infection, interferon gamma release assay

(Intern Med Advance Publication)

(DOI: 10.2169/internalmedicine.7130-21)

\section{Introduction}

Malignant peritoneal mesothelioma (MPM) is a mesenchymal tumor derived from epithelial cells. The main imaging findings are peritoneal thickening and peritoneal dissemination lesions. Differentiation from tuberculous peritonitis is important. However, if latent tuberculosis infection (LTBI) is also present, additional time is required to identify it. The examination of active tuberculosis lesions is important for differentiation.

We herein report a patient with MPM and LTBI. The early examination of active tuberculosis lesions proved useful for making the diagnosis.

\section{Case Report}

The patient was a 79-year-old man, and his medical history showed no previous tuberculosis infection. His family history included the following: his father had had pulmonary tuberculosis; and his uncle and cousin both had had stomach cancer. Although he was unemployed, he her previously run a timber carrier (20-35 years old) and managed a gas station (35-75 years old). Ascites retention was confirmed by abdominal computed tomography (CT) in March 2020. Intra- venous contrast-enhanced CT in June showed peritoneal thickening (Fig. 1A). He was suspected of having cancerous or tuberculous peritonitis.

Beginning in June 2020, he was referred to our hospital because of loss of appetite, abdominal discomfort, and weight loss. His blood sampling data were negative for CEA, CA19-9, CA125, SCC, AFP, PIVKA-II, hyaluronic acid, and PSA. sIL-2R, SYFRA, and SMRP showed a slight increase (Table). The results of an interferon gamma release assay (IGRA), called T-Spot, were positive, suggesting tuberculosis. A nucleic acid identification test for Mycobacterium tuberculosis by sputum was then performed three times, but all of the results were negative. CT performed in June showed no acinar nodule or cavities in the lung field, and pulmonary tuberculosis was negative. Intravenous contrast-enhanced CT of the abdomen in August showed exacerbation of peritoneal thickening compared to the CT findings in June, and many nodules and areas of omental thickening with a contrast-enhancing effect, which was suspected to be omental cake, were observed in the abdominal cavity (Fig. 1B, C). The volume of ascites was not sufficient to collect. Further medical scrutiny was difficult, and a laparoscopic omentum biopsy was performed in September 2020. White nodules were frequently found throughout the abdominal cavity, especially in the upper right abdomen 



Figure 1. A) Intravenous contrast-enhanced CT scans show thickening of the anterior peritoneum in June 2020 (arrowhead). B) CT scans in August 2020 show exacerbation of peritoneal thickening (arrowhead). C) CT scans of the abdomen show omental cake (arrows).

Table. Tumor Marker Laboratory Test Results.

\begin{tabular}{lclr}
\hline CEA & $1.6 \mathrm{ng} / \mathrm{mL}$ & sIL-2R & $519 \mathrm{U} / \mathrm{mL}$ \\
CA19-9 & $6 \mathrm{U} / \mathrm{mL}$ & CYFRA & $17 \mathrm{ng} / \mathrm{mL}$ \\
CA125 & $20 \mathrm{U} / \mathrm{mL}$ & HA & $72 \mathrm{ng} / \mathrm{mL}$ \\
SCC & $0.9 \mathrm{ng} / \mathrm{mL}$ & SMRP & $15.9 \mathrm{nmol} / \mathrm{L}$ \\
AFP & $3 \mathrm{ng} / \mathrm{mL}$ & PSA & $0.964 \mathrm{ng} / \mathrm{mL}$ \\
PIVKA-II & $19.35 \mathrm{mAU} / \mathrm{mL}$ & & \\
\hline
\end{tabular}

CEA: carcinoembryonic antigen, CA: carbohydrate antigen, SCC: squamous cell carcinoma, AFP: $\alpha$-fetoprotein, PIVKA-II: protein induced by vitamin $\mathrm{K}$ absence or antagonist-II, sIL-2R: soluble interleukin-2 receptor, CYFRA: cytokeratin subunit 19 fragment, HA: hyaluronic acid, SMRP: soluble mesothelin related peptides, PSA: prostate specific antigen

(Fig. 2). Part of the omentum was cut off, and surgery was completed.

A histopathological examination revealed that atypical cells with relatively abundant cytoplasm and round nuclei of different sizes were diffusely proliferated. The observation range did not contain any obvious sarcoma components. Immunostaining tests were positive for calretinin, D2-40, and WT-1 and negative for Ber-EP4 and CEA (Fig. 3). No caseous necrosis or epithelioid cell granulomas was found in the pathological tissue. Thus, the patient was diagnosed with a combination of epithelial MPM and LTBI. Systemic chemotherapy with cisplatin and pemetrexed was started in October 2020 .

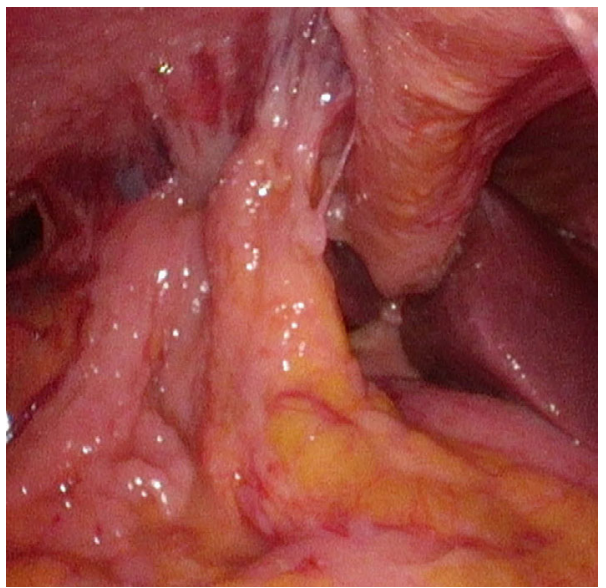

Figure 2. A laparoscopic omentum biopsy was performed. White nodules were frequently found throughout the abdominal cavity, especially in the upper right abdomen.

\section{Discussion}

We herein report a patient with MPM and LTBI. The early examination of active tuberculous lesions is important to distinguish between MPM and tuberculous peritonitis.

According to a recent review, MPM is a rare malignant tumor with 3.6 to 0.41 cases per 100,000 people $(1,2)$. The development of MPM is associated with asbestos exposure $(3,4)$. However, there was no history of asbestos expo- 


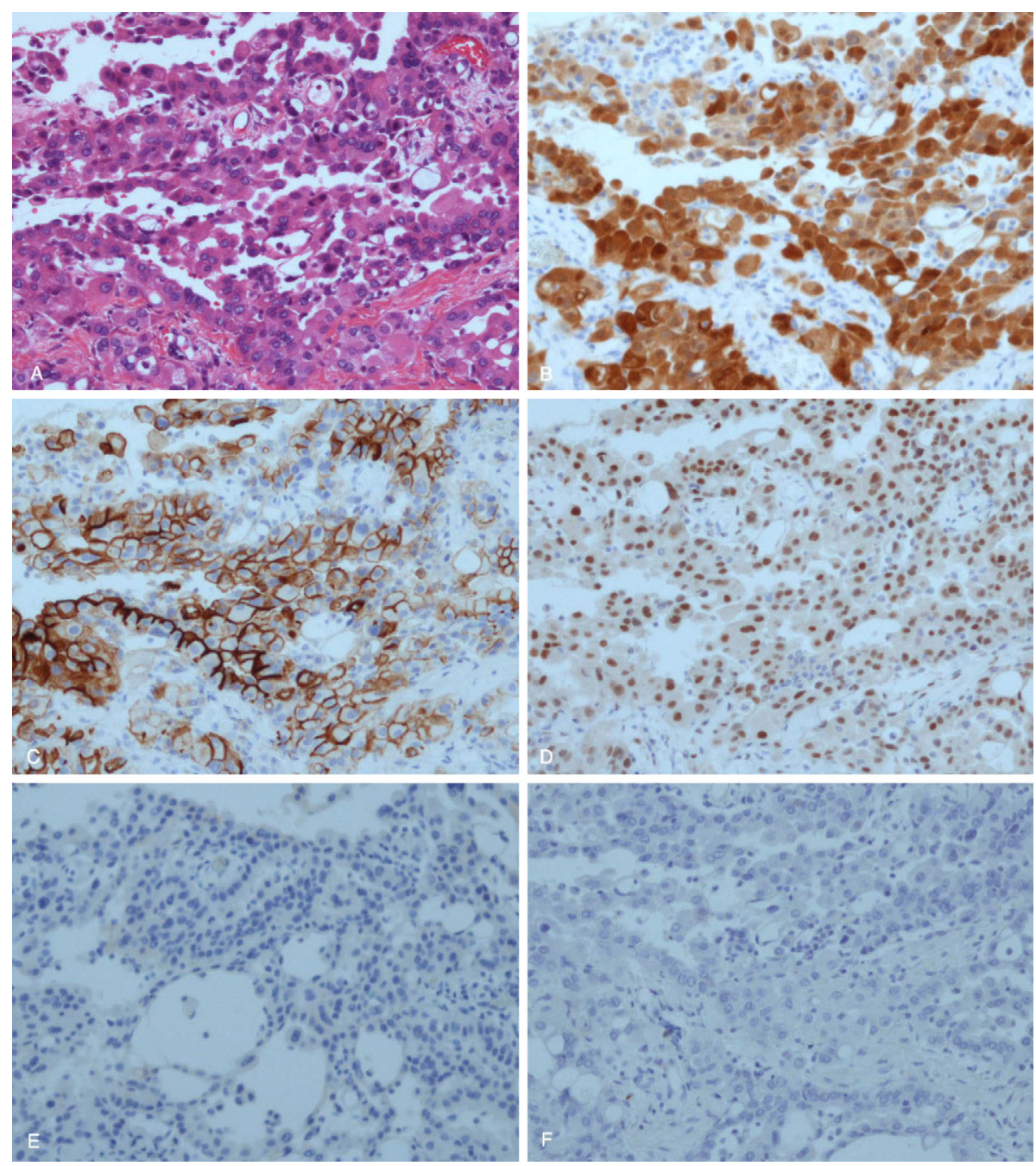

Figure 3. A) Biopsied sections of the tumor, which are stained with Hematoxylin and Eosin staining. B) The mesothelial origin of the cells is demonstrated by positive immunohistochemical staining for calretinin. C, D) Immunohistochemical staining for D2-40 and WT-1 was also positive, confirming the mesothelial origin. E, F) Immunohistochemical staining for Ber-EP4 and CEA are commonly used to identify mesothelioma, and these results were negative.

sure in this case. In addition, no pleural plaque was found on chest CT. Without treatment, the life expectancy is less than one year, so the early diagnosis and treatment initiation are critical (5). However, MPM has no specific symptoms and is often difficult to diagnose (6). In this case, a laparoscopic omentum biopsy was performed to obtain a pathological diagnosis. A CT-guided percutaneous fine-needle aspiration biopsy is a minimally invasive and safe technique (7). However, the sample amount was small, and a pathological diagnosis may not be possible. The treatment should be chosen based on the patient's age and performance status. LTBI is diagnosed when the tuberculin reaction or IGRA is positive but there are no symptoms or imaging findings of tuberculosis (8). In the present case, the patient's father had pulmonary tuberculosis, and it is possible that he had been infected with tuberculosis when he was young and recovered spontaneously. Treatment for LTBI was considered, but none was administered in order to prioritize treatment for MPM. To our knowledge, this is the first case of
MPM and LTBI, as we found no other such cases in our literature search.

The early examination of active tuberculous lesions is important for distinguishing between MPM and tuberculous peritonitis. Tuberculous peritonitis, similar to MPM, shows peritoneal thickening. It is also possible to identify tuberculous peritonitis on CT to some extent based on the findings of peritoneal and omental lesions and the presence or absence of a pleural plaque (9). However, a pathological diagnosis is required for the final diagnosis. Active tuberculosis is a known risk factor for MPM $(10,11)$. However, no studies have shown a direct link between tuberculosis and MPM. Cases of MPM and pulmonary tuberculosis have been reported (12). Active tuberculosis can be diagnosed by detecting M. tuberculosis. However, if MPM is associated with LTBI, active tuberculosis lesions need to be ruled out, and more time is required to make a diagnosis. If peritoneal thickening is present and the IGRA is positive, the presence or absence of tuberculous peritonitis or active tuberculosis 
lesions should be promptly examined. In the present case, chest CT was performed to check for the presence of active tuberculosis lesions. No acinar nodules or cavities were found in the lung field, and pulmonary tuberculosis was negative. In addition, a nucleic acid identification test for $M$. tuberculosis by sputum was performed three times, but all of the results were negative. If ascites can be collected, $M$. tuberculosis may be identified in the ascites, or M. tuberculosis can be cultivated. In this case, no ascites was found. The diagnosis of tuberculous peritonitis is often uncertain. If this is the case, diagnostic treatment may be administered. In our patient, an increase in SMRP was observed, and MPM was also a possibility; thus, an early diagnosis and treatment are desired. A laparoscopic omentum biopsy was performed without diagnostic treatment for tuberculous peritonitis to expedite a definitive diagnosis in the present case.

In our patient, MPM was associated with LTBI; however, the diseases that cause peritoneal thickening include peritonitis carcinomatosa, primary peritoneal cancer, malignant lymphoma, and desmoplastic small round-cell tumor (13-15). If LTBI is associated with these diseases, the presence or absence of active tuberculosis lesions should be investigated, similar to the present patient. In addition, if a patient is diagnosed with LTBI, treatment for LTBI may be required due to risk factors for developing active tuberculosis (16).

MPM can co-exist with LTBI. An early examination for active tuberculous infection is important to differentiate between MPM and tuberculous peritonitis. If peritoneal thickening is observed, IGRA should be performed while taking into consideration the possibility of LTBI without positively suspecting tuberculous peritonitis.

The authors state that they have no Conflict of Interest (COI).

\section{References}

1. Jiang Y, Mei Z, Cao H, et al. Meningeal metastasis of a malignant peritoneal mesothelioma: A case report and literature review. Cancer Biol Ther 20: 1409-1415, 2019.

2. Attanoos RL, Gibbs AR. Pathology of malignant mesothelioma. Histopathology 30: 403-418, 1997.

3. Mensi C, Mendola M, Dallari B, et al. Differences between perito- neal and pleural mesothelioma in Lombardy, Italy. Cancer Epidemiol 51: 68-73, 2017.

4. Sugarbaker PH, Welch LS, Mohamed F, Glehen O. A review of peritoneal mesothelioma at the Washington Cancer Institute. Surg Oncol Clin N Am 12: 605-621, xi, 2003.

5. Robinson BW, Lake RA. Advances in malignant mesothelioma. $\mathrm{N}$ Engl J Med 353: 1591-1603, 2005.

6. Boffetta P. Epidemiology of peritoneal mesothelioma: a review. Ann Oncol 18: 985-990, 2007.

7. Yu H, Zhang C, Liu S, et al. Clinical value of CT-guided percutaneous fine-needle aspiration biopsy for peritoneal lesions. BMC Med Imaging 20: 122, 2020.

8. Targeted tuberculin testing and treatment of latent tuberculosis infection. This official statement of the American Thoracic Society was adopted by the ATS Board of Directors, July 1999. This is a Joint Statement of the American Thoracic Society (ATS) and the Centers for Disease Control and Prevention (CDC). This statement was endorsed by the Council of the Infectious Diseases Society of America. (IDSA), September 1999, and the sections of this statement. Am J Respir Crit Care Med 161 (4 Pt 2): S221-S247, 2000.

9. Yin WJ, Zheng GQ, Chen YF, et al. CT differentiation of malignant peritoneal mesothelioma and tuberculous peritonitis. Radiol Med 121: 253-260, 2016.

10. Simonsen DF, Farkas DK, Søgaard M, Horsburgh CR, Sørensen HT, Thomsen RW. Tuberculosis and risk of cancer: a Danish nationwide cohort study. Int J Tuberc Lung Dis 18: 1211-1219, 2014.

11. Roviaro GC, Sartori F, Calabrò F, Varoli F. The association of pleural mesothelioma and tuberculosis. Am Rev Respir Dis 126: 569-571, 1982.

12. Kumar P, Chawla K, Khosla P, Jain S. Co-existing tuberculosis and malignant mesothelioma with multiple sites venous thrombosis: a case report. BMC Res Notes 9: 409, 2016.

13. Busch JM, Kruskal JB, Wu B. Best cases from the AFIP. Malignant peritoneal mesothelioma. Radiographics 22: 1511-1515, 2002.

14. Levy AD, Arnáiz J, Shaw JC, Sobin LH. From the archives of the AFIP: primary peritoneal tumors: imaging features with pathologic correlation. Radiographics 28: 583-607; quiz 621-582, 2008.

15. Pickhardt PJ, Bhalla S. Unusual nonneoplastic peritoneal and subperitoneal conditions: CT findings. Radiographics 25: 719-730, 2005.

16. WHO Guidelines Approved by the Guidelines Review Committee. Latent tuberculosis infection: updated and consolidated guidelines for programmatic management. World Health Organization World Health Organization 2018, Geneva, 2018.

The Internal Medicine is an Open Access journal distributed under the Creative Commons Attribution-NonCommercial-NoDerivatives 4.0 International License. To view the details of this license, please visit (https://creativecommons.org/licenses/ by-nc-nd/4.0/).

(C) The Japanese Society of Internal Medicine Intern Med Advance Publication 